\title{
COMPUTER CALCULATION OF GREEN FUNCTIONS FOR THIRD-ORDER ORDINARY DIFFERENTIAL EQUATIONS
}

\author{
I. N. Belyaeva, N. A. Chekanov, \\ L. V. Krasovskaya, and N. N. Chekanova
}

UDC 519.624.3

\begin{abstract}
In this paper, we present a method of computer calculation of Green functions in the form of generalized power series for third-order linear differential equations admitting regular singularities. For specific boundary-value problems, we construct Green functions by using the software proposed.
\end{abstract}

Keywords and phrases: ordinary differential equation, boundary-value problem, Green function, symbol-numerical computation, computer simulation.

AMS Subject Classification: $34 \mathrm{~B} 27$

1. Introduction. Green functions are very useful in solving boundary-value problems; for example, they allow one

(1) to search for solutions of inhomogeneous ordinary differential equations,

(2) to calculate eigenvalues and eigenfunctions for a given differential operator,

(3) to represent of an original boundary-value problem in the form of the Fredholm integral equation, etc. (see $[2,3,5,6]$ ).

Construction of Green functions is a very actual, but quite a difficult problem.

For constructing Green function, it is necessary to find all linearly independent solutions of the original boundary-value problem. Thus, an important problem of integrating linear ordinary differential equations arises; having solved this problem, one can then calculate the Green function of the boundary-value problem for an ordinary differential equation by computer methods. In this work, we describe solution of third-order equations by using the computer algebra system "Maple."

2. Calculation of Green functions for third-order ordinary differential equations. Consider the following third-order differential equation:

$$
p_{3}(x) y^{\prime \prime \prime}+p_{2}(x) y^{\prime \prime}+p_{1}(x) y^{\prime}+p_{0}(x) y=0 ;
$$

here the coefficients $p_{3}(x), p_{2}(x), p_{1}(x)$, and $p_{0}(x)$ are twice continuously differentiable functions on the segment $[a, b]$. For Eq. (1), we impose the boundary conditions

$$
\left\{\begin{array}{l}
\alpha_{1,0} y(a)+\alpha_{1,1} y^{\prime}(a)+\alpha_{1,2} y^{\prime \prime}(a)+\alpha_{1,3} y^{\prime \prime \prime}(a)+\beta_{1,0} y(b)+\beta_{1,1} y^{\prime}(b)+\beta_{1,2} y^{\prime \prime}(b)+\beta_{1,3} y^{\prime \prime \prime}(b)=0, \\
\alpha_{2,0} y(a)+\alpha_{2,1} y^{\prime}(a)+\alpha_{2,2} y^{\prime \prime}(a)+\alpha_{2,3} y^{\prime \prime \prime}(a)+\beta_{2,0} y(b)+\beta_{2,1} y^{\prime}(b)+\beta_{2,2} y^{\prime \prime}(b)+\beta_{2,3} y^{\prime \prime \prime}(b)=0, \\
\alpha_{3,0} y(a)+\alpha_{3,1} y^{\prime}(a)+\alpha_{3,2} y^{\prime \prime}(a)+\alpha_{3,3} y^{\prime \prime \prime}(a)+\beta_{3,0} y(b)+\beta_{3,1} y^{\prime}(b)+\beta_{3,2} y^{\prime \prime}(b)+\beta_{3,3} y^{\prime \prime \prime}(b)=0,
\end{array}\right.
$$

where $\alpha_{i, k}, \beta_{i, k}, i=1,2,3, k=0,1,2,3$, are coefficients such that

$$
\sum_{i} \sum_{k}\left(\alpha_{i, k}^{2}+\beta_{i, k}^{2}\right) \neq 0 \text {. }
$$

Assume that the problem (1) with the boundary conditions (2) has only the trivial solution in the class of twice continuously differentiable functions. However, if we reject the continuity condition for

Translated from Itogi Nauki i Tekhniki, Seriya Sovremennaya Matematika i Ee Prilozheniya. Tematicheskie Obzory, Vol. 165, Proceedings of the IV International Scientific Conference "Actual Problems of Applied Mathematics," Kabardino-Balkar Republic, Nalchik, Elbrus Region, May 22-26, 2018. Part I, 2019. 
the second derivative at a point $x=\xi, \xi \in[a, b]$, then there exists a nonzero solution $G(x, \xi)$ called the Green function.

To construct the Green function for the boundary-value problem (1) with the conditions (2), we first must solve the Cauchy problem at the point $x_{0}$. We search for three linearly independent solutions of Eq. (1) in the form (see [1])

$$
\begin{aligned}
& y_{1}(x)=1+\sum_{k=3}^{N} c_{k}^{(1)}\left(x-x_{0}\right)^{k}, \\
& y_{2}(x)=\left(x-x_{0}\right)+\sum_{k=3}^{N} c_{k}^{(2)}\left(x-x_{0}\right)^{k}, \\
& y_{3}(x)=\left(x-x_{0}\right)^{2} / 2+\sum_{k=3}^{N} c_{k}^{(3)}\left(x-x_{0}\right)^{k},
\end{aligned}
$$

where $c_{k}^{(1)}, c_{k}^{(2)}$, and $c_{k}^{(3)}$ are numerical coefficients. Then the Green function has the form:

$$
G(x, \xi)=\left\{\begin{array}{ll}
G_{L}(x, \xi), & a \leq x \leq \xi \leq b, \\
G_{R}(x, \xi), & a \leq \xi \leq x \leq b,
\end{array} \quad G_{L}(x, \xi)=\sum_{k=1}^{3}\left[A_{k}(\xi)+B_{k}(\xi)\right] y_{k}(x),\right.
$$

Using the basic properties of Green functions, we find the unknown functional coefficients $A_{k}(\xi)$ and $B_{k}(\xi)$.

1. The Green function and its first derivative are continuous at the point $x=\xi$.

2. At the point $x=\xi$, the second derivative of the Green function has a jump discontinuity:

$$
\left.\frac{\partial^{2} G(x, \xi)}{\partial x^{2}}\right|_{x=\xi+0}-\left.\frac{\partial^{2} G(x, \xi)}{\partial x^{2}}\right|_{x=\xi-0}=\frac{1}{p_{3}(\xi)} .
$$

3. The Green function satisfies the differential equation (1).

4. The Green function satisfies the boundary conditions (2).

A theorem from the theory of differential equations (see, e.g., $[4,7]$ ) states that if the boundaryvalue problem (1) with the conditions (2) has only trivial solution $y(x) \equiv 0$, then there exists a unique Green function $G(x, \xi)$.

Note that, since we search for a solution of the Cauchy problem if the power-series form, the Green function $G(x, \xi)$ will be also obtained in this form.

From the properties 1 and 2 of the Green function we obtain the following system of algebraic equations:

$$
\sum_{k=1}^{3} B_{k}(\xi) \cdot y_{k}(\xi)=0, \quad \sum_{k=1}^{3} B_{k}(\xi) \cdot y_{k}^{\prime}(\xi)=0, \quad \sum_{k=1}^{3} B_{k}(\xi) \cdot y_{k}^{\prime \prime}(\xi)=-\frac{1}{2 p_{3}(\xi)}
$$

Solving the system (6), we find the coefficients $B_{k}(\xi), k=1,2,3$. Note that the determinant of this system is the Wronskian of the linearly independent solutions $y_{1}, y_{2}, y_{3}$ :

$$
W(\xi) \equiv W\left[y_{1}, y_{2}, y_{3}\right]=\left|\begin{array}{lll}
y_{1} & y_{2} & y_{3} \\
y_{1}^{\prime} & y_{2}^{\prime} & y_{3}^{\prime} \\
y_{1}^{\prime \prime} & y_{2}^{\prime \prime} & y_{3}^{\prime \prime}
\end{array}\right| \neq 0
$$


Therefore, the system of algebraic equations (6) possesses a unique solution $B_{k}(\xi), k=1,2,3$. Further, we calculate the functional coefficients $A_{k}(\xi), k=1,2,3$. Taking into account the boundary conditions (2), we arrive at the following system of three equations:

$$
\begin{aligned}
& A_{1}(\xi)\left[\alpha_{1,0} y_{1}(a)+\alpha_{1,1} y_{1}^{\prime}(a)+\alpha_{1,2} y_{1}^{\prime \prime}(a)+\alpha_{1,3} y_{1}^{\prime \prime \prime}(a)+\beta_{1,0} y_{1}(b)+\beta_{1,1} y_{1}^{\prime}(b)+\beta_{1,2} y_{1}^{\prime \prime}(b)+\beta_{1,3} y_{1}^{\prime \prime \prime}(b)\right]+ \\
& +A_{2}(\xi)\left[\alpha_{1,0} y_{1}(a)+\alpha_{1,1} y_{1}^{\prime}(a)+\alpha_{1,2} y_{1}^{\prime \prime}(a)+\alpha_{1,3} y_{1}^{\prime \prime \prime}(a)+\beta_{1,0} y_{1}(b)+\beta_{1,1} y_{1}^{\prime}(b)+\beta_{1,2} y_{1}^{\prime \prime}(b)+\beta_{1,3} y_{1}^{\prime \prime \prime}(b)\right]+ \\
& +A_{3}(\xi)\left[\alpha_{1,0} y_{1}(a)+\alpha_{1,1} y_{1}^{\prime}(a)+\alpha_{1,2} y_{1}^{\prime \prime}(a)+\alpha_{1,3} y_{1}^{\prime \prime \prime}(a)+\beta_{1,0} y_{1}(b)+\beta_{1,1} y_{1}^{\prime}(b)+\beta_{1,2} y_{1}^{\prime \prime}(b)+\beta_{1,3} y_{1}^{\prime \prime \prime}(b)\right]= \\
& =B_{1}(\xi)\left[\alpha_{1,0} y_{1}(a)+\alpha_{1,1} y_{1}^{\prime}(a)+\alpha_{1,2} y_{1}^{\prime \prime}(a)+\alpha_{1,3} y_{1}^{\prime \prime \prime}(a)-\beta_{1,0} y_{1}(b)-\beta_{1,1} y_{1}^{\prime}(b)-\beta_{1,2} y_{1}^{\prime \prime}(b)-\beta_{1,3} y_{1}^{\prime \prime \prime}(b)\right]+ \\
& +B_{2}(\xi)\left[\alpha_{1,0} y_{1}(a)+\alpha_{1,1} y_{1}^{\prime}(a)+\alpha_{1,2} y_{1}^{\prime \prime}(a)+\alpha_{1,3} y_{1}^{\prime \prime \prime}(a)-\beta_{1,0} y_{1}(b)-\beta_{1,1} y_{1}^{\prime}(b)-\beta_{1,2} y_{1}^{\prime \prime}(b)-\beta_{1,3} y_{1}^{\prime \prime \prime}(b)\right]+ \\
& +B_{3}(\xi)\left[\alpha_{1,0} y_{1}(a)+\alpha_{1,1} y_{1}^{\prime}(a)+\alpha_{1,2} y_{1}^{\prime \prime}(a)+\alpha_{1,3} y_{1}^{\prime \prime \prime}(a)-\beta_{1,0} y_{1}(b)-\beta_{1,1} y_{1}^{\prime}(b)-\beta_{1,2} y_{1}^{\prime \prime}(b)-\beta_{1,3} y_{1}^{\prime \prime \prime}(b)\right] \text {, } \\
& A_{1}(\xi)\left[\alpha_{2,0} y_{1}(a)+\alpha_{2,1} y_{1}^{\prime}(a)+\alpha_{2,2} y_{1}^{\prime \prime}(a)+\alpha_{2,3} y_{1}^{\prime \prime \prime}(a)+\beta_{2,0} y_{1}(b)+\beta_{2,1} y_{1}^{\prime}(b)+\beta_{2,2} y_{1}^{\prime \prime}(b)+\beta_{2,3} y_{1}^{\prime \prime \prime}(b)\right]+ \\
& +A_{2}(\xi)\left[\alpha_{2,0} y_{1}(a)+\alpha_{2,1} y_{1}^{\prime}(a)+\alpha_{2,2} y_{1}^{\prime \prime}(a)+\alpha_{2,3} y_{1}^{\prime \prime \prime}(a)+\beta_{1,0} y_{1}(b)+\beta_{2,1} y_{1}^{\prime}(b)+\beta_{2,2} y_{1}^{\prime \prime}(b)+\beta_{2,3} y_{1}^{\prime \prime \prime}(b)\right]+ \\
& +A_{3}(\xi)\left[\alpha_{2,0} y_{1}(a)+\alpha_{2,1} y_{1}^{\prime}(a)+\alpha_{2,2} y_{1}^{\prime \prime}(a)+\alpha_{2,3} y_{1}^{\prime \prime \prime}(a)+\beta_{2,0} y_{1}(b)+\beta_{2,1} y_{1}^{\prime}(b)+\beta_{2,2} y_{1}^{\prime \prime}(b)+\beta_{2,3} y_{1}^{\prime \prime \prime}(b)\right]= \\
& =B_{1}(\xi)\left[\alpha_{2,0} y_{1}(a)+\alpha_{2,1} y_{1}^{\prime}(a)+\alpha_{2,2} y_{1}^{\prime \prime}(a)+\alpha_{2,3} y_{1}^{\prime \prime \prime}(a)-\beta_{2,0} y_{1}(b)-\beta_{2,1} y_{1}^{\prime}(b)-\beta_{2,2} y_{1}^{\prime \prime}(b)-\beta_{2,3} y_{1}^{\prime \prime \prime}(b)\right]+ \\
& +B_{2}(\xi)\left[\alpha_{2,0} y_{1}(a)+\alpha_{2,1} y_{1}^{\prime}(a)+\alpha_{2,2} y_{1}^{\prime \prime}(a)+\alpha_{2,3} y_{1}^{\prime \prime \prime}(a)-\beta_{2,0} y_{1}(b)-\beta_{2,1} y_{1}^{\prime}(b)-\beta_{2,2} y_{1}^{\prime \prime}(b)-\beta_{2,3} y_{1}^{\prime \prime \prime}(b)\right]+ \\
& +B_{3}(\xi)\left[\alpha_{2,0} y_{1}(a)+\alpha_{2,1} y_{1}^{\prime}(a)+\alpha_{2,2} y_{1}^{\prime \prime}(a)+\alpha_{2,3} y_{1}^{\prime \prime \prime}(a)-\beta_{2,0} y_{1}(b)-\beta_{2,1} y_{1}^{\prime}(b)-\beta_{2,2} y_{1}^{\prime \prime}(b)-\beta_{2,3} y_{1}^{\prime \prime \prime}(b)\right] \text {, } \\
& A_{1}(\xi)\left[\alpha_{3,0} y_{1}(a)+\alpha_{3,1} y_{1}^{\prime}(a)+\alpha_{3,2} y_{1}^{\prime \prime}(a)+\alpha_{3,3} y_{1}^{\prime \prime \prime}(a)+\beta_{3,0} y_{1}(b)+\beta_{3,1} y_{1}^{\prime}(b)+\beta_{3,2} y_{1}^{\prime \prime}(b)+\beta_{3,3} y_{1}^{\prime \prime \prime}(b)\right]+ \\
& +A_{2}(\xi)\left[\alpha_{3,0} y_{1}(a)+\alpha_{3,1} y_{1}^{\prime}(a)+\alpha_{3,2} y_{1}^{\prime \prime}(a)+\alpha_{3,3} y_{1}^{\prime \prime \prime}(a)+\beta_{3,0} y_{1}(b)+\beta_{3,1} y_{1}^{\prime}(b)+\beta_{3,2} y_{1}^{\prime \prime}(b)+\beta_{3,3} y_{1}^{\prime \prime \prime}(b)\right]+ \\
& +A_{3}(\xi)\left[\alpha_{3,0} y_{1}(a)+\alpha_{3,1} y_{1}^{\prime}(a)+\alpha_{3,2} y_{1}^{\prime \prime}(a)+\alpha_{3,3} y_{1}^{\prime \prime \prime}(a)+\beta_{3,0} y_{1}(b)+\beta_{3,1} y_{1}^{\prime}(b)+\beta_{3,2} y_{1}^{\prime \prime}(b)+\beta_{3,3} y_{1}^{\prime \prime \prime}(b)\right]= \\
& =B_{1}(\xi)\left[\alpha_{3,0} y_{1}(a)+\alpha_{3,1} y_{1}^{\prime}(a)+\alpha_{3,2} y_{1}^{\prime \prime}(a)+\alpha_{3,3} y_{1}^{\prime \prime \prime}(a)-\beta_{3,0} y_{1}(b)-\beta_{3,1} y_{1}^{\prime}(b)-\beta_{3,2} y_{1}^{\prime \prime}(b)-\beta_{3,3} y_{1}^{\prime \prime \prime}(b)\right]+ \\
& +B_{2}(\xi)\left[\alpha_{3,0} y_{1}(a)+\alpha_{3,1} y_{1}^{\prime}(a)+\alpha_{3,2} y_{1}^{\prime \prime}(a)+\alpha_{3,3} y_{1}^{\prime \prime \prime}(a)-\beta_{3,0} y_{1}(b)-\beta_{3,1} y_{1}^{\prime}(b)-\beta_{3,2} y_{1}^{\prime \prime}(b)-\beta_{3,3} y_{1}^{\prime \prime \prime}(b)\right]+ \\
& +B_{3}(\xi)\left[\alpha_{3,0} y_{1}(a)+\alpha_{3,1} y_{1}^{\prime}(a)+\alpha_{3,2} y_{1}^{\prime \prime}(a)+\alpha_{3,3} y_{1}^{\prime \prime \prime}(a)-\beta_{3,0} y_{1}(b)-\beta_{3,1} y_{1}^{\prime}(b)-\beta_{3,2} y_{1}^{\prime \prime}(b)-\beta_{3,3} y_{1}^{\prime \prime \prime}(b)\right] \text {. }
\end{aligned}
$$

Substituting the functional coefficients $A_{k}(\xi)$ and $B_{k}(\xi), k=1,2,3$, into the formula (4), we obtain the Green function in the analytic form.

\section{Algorithm of the search for Green functions for third-order ordinary differential equations. \\ Input:}

- $p_{3}(x), p_{2}(x), p_{1}(x), p_{0}(x)$ are functional coefficients in the third-order ordinary differential equation $(1)$,

- $N$ is the number of terms of the power series (3),

- $a$ and $b$ are the boundary points of the segment on which the Green function $G_{L}(x, \xi)$ will be constructed,

- $\alpha_{1,0}, \alpha_{1,1}, \alpha_{1,2}, \alpha_{1,3}, \alpha_{2,0}, \alpha_{2,1}, \alpha_{2,2}, \alpha_{2,3}, \alpha_{3,0}, \alpha_{3,1}, \alpha_{3,2}, \alpha_{3,3}, \beta_{1,0}, \beta_{1,1}, \beta_{1,2}, \beta_{1,3}, \beta_{2,0}, \beta_{2,1}$, $\beta_{2,2}, \beta_{2,3}, \beta_{3,0}, \beta_{3,1}, \beta_{3,2}, \beta_{3,3}$ are numerical coefficients for the boundary-value problem in the conditions (2).

\section{Output:}

- $y_{1}(x), y_{2}(x), y_{3}(x)$ are linearly independent solutions of the given third-order ordinary differential equation (1),

- $G_{L}(x, \xi)$ is the Green function on the segment $[a, b]$, where $a \leq x \leq \xi \leq b$,

- $G_{R}(x, \xi)$ is the Green function on the segment $[a, b]$, where $a \leq \xi \leq x \leq b$.

\section{Description of the algorithm:}


1. Construction of three linearly independent solutions of the third-order differential equation in the generalized power-series form (see [1]).

2. Verification of the solutions constructed.

3. Solution of the system of algebraic equations (6) for the functional coefficients $B_{k}(\xi), k=1,2,3$.

4. Verification of the functional coefficients $B_{k}(\xi), k=1,2,3$.

5. Solution of the system of algebraic equations (8), (9), (10) for the coefficients $A_{k}(\xi), k=1,2,3$.

6. Verification of the coefficients $A_{k}(\xi), k=1,2,3$.

7. Construction of the Green functions $G_{L}(x, \xi)$ under the condition $a \leq x \leq \xi \leq b$ by the formula (4).

8. Construction of the Green function $G_{R}(x, \xi)$ under the condition $a \leq \xi \leq x \leq b$ by the formula 4 .

9. Verification of the four basic properties of the Green function $G(x, \xi)$.

4. Computer simulation of Green functions. The software developed was tested on the differential equation

$$
y^{\prime \prime \prime}-6 y^{\prime \prime}+11 y^{\prime}-6 y=0
$$

with the boundary conditions

$$
y(0)=0, \quad y(1)=0, \quad y^{\prime \prime}(0)=0 .
$$

First, we construct the Green function for this boundary-value problem without using a computer. The fundamental system of solutions of Eq. (11) with the conditions (12) has the form

$$
y_{1}=e^{x}, \quad y_{2}=e^{2 x}, \quad y_{2}=e^{3 x} .
$$

The function $G(x, \xi)$ can be constructed from the solutions (13) by the algorithm proposed above. Taking into account the basic properties 1 and 2 of the Green function, we obtain the system

$$
\left\{\begin{array}{l}
B_{1}(\xi) \cdot y_{1}(\xi)+B_{2}(\xi) \cdot y_{2}(\xi)+B_{3}(\xi) \cdot y_{3}(\xi)=0 \\
B_{1}(\xi) \cdot y_{1}^{\prime}(\xi)+B_{2}(\xi) \cdot y_{2}^{\prime}(\xi)+B_{3}(\xi) \cdot y_{3}^{\prime}(\xi)=0 \\
B_{1}(\xi) \cdot y_{1}^{\prime \prime}(\xi)+B_{2}(\xi) \cdot y_{2}^{\prime \prime}(\xi)+B_{3}(\xi) \cdot y_{3}^{\prime \prime}(\xi)=-\frac{1}{2 p_{3}(\xi)}
\end{array}\right.
$$

Since $p_{3}(\xi) \neq 0$, the system (14) is always solvable and has a unique solution. Thus, the principal determinant of the system (14) is the Wronskian $W\left[y_{1}, y_{2}, y_{3}\right] \neq 0$. Solving the system (14), we find the coefficients $B_{k}(\xi), k=1,2,3$ :

$$
B_{1}(\xi)=-\frac{1}{4} e^{-\xi}, \quad B_{2}(\xi)=\frac{1}{2} e^{-2 \xi}, \quad B_{3}(\xi)=-\frac{1}{4} e^{-3 \xi} .
$$

Further, taking into account the boundary conditions (12), we obtain the system of equations

$$
\left\{\begin{array}{c}
A_{1}(\xi)+A_{2}(\xi)+A_{3}(\xi)=-B_{1}(\xi)-B_{2}(\xi)-B_{3}(\xi) \\
A_{1}(\xi)+4 A_{2}(\xi)+9 A_{3}(\xi)=-B_{1}(\xi)-4 B_{2}(\xi)-9 B_{3}(\xi), \\
A_{1}(\xi)+e A_{2}(\xi)+e^{2} A_{3}(\xi)=B_{1}(\xi)+e B_{2}(\xi)+e^{2} B_{3}(\xi) .
\end{array}\right.
$$

Solving this system, we obtain the coefficients $A_{k}(\xi), k=1,2,3$ :

$$
\begin{aligned}
& A_{1}(\xi)=-\frac{1}{4\left(3 e^{2}-8 e+5\right)}\left(-20 e^{1+\xi}+5 e^{2 \xi}+10 e^{2}+8 e^{1-2 \xi}-3 e^{2+2 \xi}\right) e^{-3 \xi}, \\
& A_{2}(\xi)=\frac{1}{2\left(3 e^{2}-8 e+5\right)}\left(-8 e^{1+\xi}+8 e^{2 \xi}+8 e^{2}-5 e^{\xi}-3 e^{2+\xi}\right) e^{-3 \xi} \\
& A_{3}(\xi)=-\frac{1}{4\left(3 e^{2}-8 e+5\right)}\left(-12 e^{1+\xi}-5+6 e^{2 \xi}+3 e^{2}+8 e\right) e^{-3 \xi}
\end{aligned}
$$


Substituting the functions $B_{k}(\xi)$ and $A_{k}(\xi), k=1,2,3$, into (4), we obtain the exact Green function for the problem (11) with the conditions (12) in the form

$$
\begin{gathered}
G(x, \xi)= \begin{cases}G_{L}(x, \xi), & 0 \leq x \leq \xi \leq 1, \\
G_{R}(x, \xi), & 0 \leq x \leq \xi \leq 1,\end{cases} \\
G_{L}(x, \xi)=\frac{e^{x}\left(-5 / 2 e^{-\xi}+5 e^{1-2 \xi}-5 / 2 e^{2-3 \xi}\right)}{3 e^{2}-8 e+5}+\frac{e^{2 x}\left(4 e^{-\xi}-8 e^{1-2 \xi}+4 e^{2-3 \xi}\right)}{3 e^{2}-8 e+5}+ \\
+\frac{e^{3 x}\left(-3 / 2 e^{-\xi}+3 e^{1-2 \xi}-3 / 2 e^{2-3 \xi}\right)}{3 e^{2}-8 e+5}, \\
G_{R}(x, \xi)=\frac{e^{x}\left(3 / 2 e^{2-\xi}-4 e^{1-\xi}+5 e^{1-2 \xi}-5 / 2 e^{2-3 \xi}\right)}{3 e^{2}-8 e+5}+\frac{e^{2 x}\left(4 e^{-\xi}-3 e^{2-2 \xi}-5 e^{-2 \xi}+4^{2-3 \xi}\right)}{3 e^{2}-8 e+5}+ \\
+\frac{e^{3 x}\left(-3 / 2 e^{-\xi}+3 e^{1-2 \xi}+5 / 2 e^{-3 \xi}-4 e^{1-3 \xi}\right)}{3 e^{2}-8+5}
\end{gathered}
$$

Using the software for the Maple computer algebra system, we computed an approximate Green function (with $N=6$ ) for the boundary-value problem (11) under the conditions (12):

$$
\begin{aligned}
& G(x, \xi)=\left\{\begin{array}{l}
G_{L}^{6}(x, \xi), \quad 0 \leq x \leq \xi \leq 1, \\
G_{R}^{6}(x, \xi), \quad 0 \leq \xi \leq x \leq 1,
\end{array}\right. \\
& G_{L}^{6}(x, \xi)= \frac{19663}{35489} x-\frac{82489}{35489} x \xi+\frac{308167}{70978} x \xi^{2}-\frac{216293}{212934} x^{3}-\frac{1059601}{212934} x \xi^{3}+\frac{907379}{212934} x^{3} \xi-\frac{98315}{70978} x^{4}+ \\
&+ \frac{3462703}{851736} x \xi^{4}-\frac{3389837}{425868} x^{3} \xi^{2}+\frac{412445}{70978} x^{4} \xi-\frac{4699457}{4258680} x^{5}-\frac{10969609}{4258680} x \xi^{5}+\frac{11655611}{1277604} x^{3} \xi^{3}- \\
&- \frac{1540835}{141956} x^{4} \xi^{2}+\frac{19714871}{4258680} x^{5} \xi-\frac{137641}{212934} x^{6}+\frac{46783051}{12776040} x \xi^{6}-\frac{38089733}{5110416} x^{3} \xi^{4}+ \\
&+ \frac{5298005}{425868} x^{4} \xi^{3}-\frac{73651913}{8517360} x^{5} \xi^{2}+\frac{577423}{212934} x^{6} \xi+\frac{120665699}{25552080} x^{3} \xi^{5}-\frac{17313515}{1703472} x^{4} \xi^{4}+ \\
&+ \frac{253244639}{25552080} x^{5} \xi^{3}-\frac{2157169}{425868} x^{6} \xi^{2}-\frac{514613561}{76656240} x^{3} \xi^{6}+\frac{10969609}{1703472} x^{2} \xi^{5}-\frac{827586017}{102208320} x^{5} \xi^{4}+ \\
&+ \frac{7417207}{1277604} x^{6} \xi^{3}-\frac{46783051}{5110416} x^{4} \xi^{6}+\frac{2621736551}{511041600} x^{5} \xi^{5}-\frac{24238921}{5110416} x^{6} \xi^{4}-\frac{11181149189}{1533124800} x^{5} \xi^{6}+ \\
&+ \frac{76787263}{25552080} x^{6} \xi^{5}-\frac{327481357}{76656240} x^{6} \xi^{6}, \\
& G_{R}^{6}(x, \xi)= \frac{19663}{35489} x+\frac{1}{2} \xi^{2}-\frac{117978}{35489} x \xi+\frac{1}{2} x^{2}-\xi^{3}+\frac{521101}{70978} x \xi^{2}-3 x^{2} \xi-\frac{3359}{212934} x^{3}-\frac{25}{24} \xi^{4}+ \\
&+ \frac{324471}{35489} x \xi^{3}+\frac{25}{4} x^{2} \xi^{2}+\frac{3359}{35489} x^{3} \xi-\frac{292555}{851736} x^{4}-\frac{3}{4} \xi^{5}+\frac{6656713}{851736} x \xi^{4}-\frac{15}{2} x^{2} \xi^{3}- \\
&-\frac{195827}{425868} x^{3} \xi^{2}+\frac{292555}{141956} x^{4} \xi-\frac{1505447}{4258680} x^{5}+\frac{301}{720} \xi^{6}-\frac{3608633}{709780} x \xi^{5}+\frac{301}{48} x^{2} \xi^{4}+ \\
&+\frac{54079}{70978} x^{3} \xi^{3}-\frac{7807831}{1703472} x^{4} \xi^{2}+\frac{1505447}{709780} x^{5} \xi-\frac{5834731}{25552080} x^{6}+\frac{31962119}{6388020} x \xi^{6}- \\
&-\frac{161}{40} x^{2} \xi^{5}-\frac{3807359}{5110416} x^{3} \xi^{4}+\frac{1627427}{283912} x^{4} \xi^{3}-\frac{39369539}{8517360} x^{5} \xi^{2}-\frac{5834731}{4258680} x^{6} \xi+\frac{605}{144} x^{2} \xi^{6}+ \\
&+ 3 \xi^{5}-\frac{100407955}{20441664} x^{4} \xi^{4}+\frac{8105023}{1419560} x^{5} \xi^{3}-\frac{30301211}{10220832} x^{6} \xi^{2}-\frac{26994701}{76656240} x^{3} \xi^{6} .
\end{aligned}
$$

Comparison of the approximate Green function (19) constructed by using the method proposed above with the exact Green function calculated by the formula (18) shows that the approximate Green 
function $G_{\text {calc }}$ constructed by $N=13$ terms of series differs from the exact Green function $G_{\text {theor }}$ only by $1.3 \%$. Increasing the number of terms leads to decreasing the error; for example, if $N=16$, then the relative error becomes $0.003 \%$.

We also note that the error depends on $\xi$. Calculations with $N=13$ show that the absolute error $\Delta=\left|G_{\text {theor }}-G_{\text {calc }}\right|$ for $\xi=0.25$ is equal to $\Delta=8 \cdot 10^{-7}$, whereas for $\xi=0.5$ we have $\Delta=1.5 \cdot 10^{-6}$ and for $\xi=0.75$ we have $\Delta=0.00015$. The relative error $\varepsilon=\Delta / G_{\text {theor }} \cdot 100 \%$ is equal to $\varepsilon=0.038 \%$ for $\xi=0.25$. If $\xi=0.5$, then $\varepsilon=1.3 \%$; if $\xi=0.75$, then $\varepsilon=3 \%$.

We also calculate the absolute error for $N=16$. In this case, we have $\Delta=6 \cdot 10^{-9}$ for $\xi=0.25$, $\Delta=8 \cdot 10^{-9}$ for $\xi=0.5$, and $\Delta=6 \cdot 10^{-7}$ for $\xi=0.75$. The relative error is $\varepsilon=4 \cdot 10^{-5} \%$ for $\xi=0.25$, $\varepsilon=0.003 \%$ for $\xi=0.5$, and $\varepsilon=0.006 \%$ for $\xi=0.75$.

Consider the differential equation

$$
y^{\prime \prime \prime}=0
$$

with the boundary conditions

$$
y(0)=0, \quad y(1)=0, \quad y^{\prime}(0)=y^{\prime}(1) .
$$

Using the software developed, we obtain the Green function

$$
G(x, \xi)= \begin{cases}G_{L}(x, \xi), & 0 \leq x \leq \xi \leq 1 \\ G_{R}(x, \xi), & 0 \leq \xi \leq x \leq 1\end{cases}
$$

where

$$
G_{L}=\frac{1}{2}\left(x^{2} \xi-x \xi^{2}-x^{2}+x \xi\right), \quad G_{R}=\frac{1}{2}\left(x^{2} \xi-x \xi^{2}-x \xi+\xi^{2}\right) .
$$

The expression (22) coincides with the exact formula; it is anti-self-adjoint (see $[5,6]$ ).

Now we consider the same differential equation (20) with the other boundary conditions:

$$
y(0)=0, \quad y(1)=0, \quad y^{\prime}(0)+y^{\prime}(1)=0 .
$$

This equation has a nontrivial solution; therefore, the Green function $G(x, \xi)$ does nor exist.

Further, for the differential equation (20) with the boundary conditions

$$
y(0)=0, \quad y^{\prime}(1)=0, \quad y^{\prime}(0)=y(1),
$$

we obtain the following Green function:

$$
G(x, \xi)= \begin{cases}G_{L}(x, \xi), & 0 \leq x \leq \xi \leq 1 \\ G_{R}(x, \xi), & 0 \leq \xi \leq x \leq 1\end{cases}
$$

where

$$
G_{L}=-\frac{1}{2} x^{2} \xi^{2}+x^{2} \xi+x \xi^{2}-\frac{1}{2} x^{2}-x \xi, \quad G_{R}=-\frac{1}{2} x^{2} \xi^{2}+x^{2} \xi+x \xi^{2}-2 x \xi+\frac{1}{2} \xi^{2} .
$$

Note that in this case, the Green function $G(x, \xi)$ defined by the formula (25) is not anti-self-adjoint.

The boundary-value problems discussed above demonstrate the importance of boundary conditions.

Consider the differential equation

$$
y^{\prime \prime \prime}-x^{2} y^{\prime \prime}+(a+b-1) x y^{\prime}-a b y=0
$$

where $a$ and $b$ are parameters, with the boundary conditions

$$
y(0)=0, \quad y(1)=0, \quad y^{\prime}(0)=y^{\prime}(1) .
$$


Using the software developed, we construct the Green function in the power-series form with $N=5$. For simplicity, we set $a=b=1$; then the Green function has the form

$$
\begin{gathered}
G(x, \xi)=\left\{\begin{array}{l}
G_{L}(x, \xi), \quad 0 \leq x \leq \xi \leq 1, \\
G_{R}(x, \xi), \quad 0 \leq \xi \leq x \leq 1,
\end{array}\right. \\
G_{L}=-\frac{1}{120} x^{5}+\frac{1}{3} x^{2} \xi^{3}-\frac{24}{125} x \xi^{4}-\frac{3}{25} x^{2} \xi^{2}+\frac{12}{25} x^{2} \xi-\frac{173}{375} x \xi^{2}-\frac{1}{2} x^{2}+\frac{64}{125} x \xi, \\
G_{R}=\frac{1}{12} x^{3} \xi^{2}+\frac{183}{1000} x \xi^{4}-\frac{2}{15} \xi^{5}-\frac{3}{25} x^{2} \xi^{2}+\frac{12}{25} x^{2} \xi-\frac{173}{375} x \xi^{2}-\frac{61}{125} x \xi+\frac{1}{2} \xi^{2} . \\
\text { REFERENCES }
\end{gathered}
$$

1. I. N. Belyaeva and N. A. Chekanov, Symbolic-numerical integration of a third-order linear differential equation in the form of generalized series, Certificate of state registration of a computer program No. 2010614257 (2010).

2. E. L. Ince, Ordinary Differential Equations, Dover, New York (1944).

3. E. Kamke, Differentialgleichungen. Lösungsmethoden und Lösungen. I. Gewöhnliche Differentialgleichungen, B. G. Teubner, Stuttgart (1977).

4. M. L. Krasnov, A. I. Kiselev, G. I. Makarenko, Integral Equations [in Russian], Nauka, Moscow (1976).

5. G. Sansone, Equazioni Differenziali nel Campo Reale. Vol. 1, Bologna (1948).

6. G. Sansone, Equazioni Differenziali nel Campo Reale. Vol. 2, Bologna (1949).

7. F. G. Tricomi, Differential Equations, Blackie \& Son, London (1961).

I. N. Belyaeva

Belgorod National Research University

E-mail: ibelyaeva@bsu.edu.ru

N. A. Chekanov

Belgorod National Research University

E-mail: Chekanov@bsu.edu.ru

L. V. Krasovskaya

Belgorod National Research University

E-mail: krasovskaya@bsu.edu.ru

N. N. Chekanova

Banking University, Kharkiv, Ukraine

E-mail: chekanova76@list.ru 